PROCEEDINGS OF THE

AMERICAN MATHEMATICAL SOCIETY

Volume 126, Number 10, October 1998, Pages 2949-2955

S 0002-9939(98)04814-X

\title{
SEMIGROUP REPRESENTATIONS, POSITIVE DEFINITE FUNCTIONS AND ABELIAN $C^{*}$-ALGEBRAS
}

\author{
P. RESSEL AND W. J. RICKER
}

(Communicated by Palle E. T. Jorgensen)

\begin{abstract}
It is shown that every *-representation of a commutative semigroup $S$ with involution via operators on a Hilbert space has an integral representation with respect to a unique, compactly supported, selfadjoint Radon spectral measure defined on the Borel sets of the character space of $S$. The main feature is that the proof, which is based on the theory of positive definite functions, makes no use what-so-ever (directly or indirectly) of the theory of $C^{*}$-algebras or more general Banach algebra arguments. Accordingly, this integral representation theorem is used to give a new proof of the GelfandNaimark theorem for abelian $C^{*}$-algebras.
\end{abstract}

Let $H$ be a Hilbert space and $\mathcal{A} \subseteq \mathcal{L}(H)$ an abelian $C^{*}$-algebra, that is, the identity operator $I$ belongs to $\mathcal{A}$, the adjoint operator $T^{*} \in \mathcal{A}$ whenever $T \in \mathcal{A}$, and the (commutative) algebra $\mathcal{A}$ is closed for the operator norm topology in the space $\mathcal{L}(H)$ of all continuous linear operators of $H$ into itself. The Gelfand-Naimark theorem can be formulated as follows; see [5, Theorem 12.22] for a very readable account of this result.

Theorem 1. Let $H$ be a Hilbert space and $\mathcal{A} \subseteq \mathcal{L}(H)$ an abelian $C^{*}$-algebra. Then there exists a unique selfadjoint, Radon spectral measure $F: \mathcal{B}(\Delta) \rightarrow \mathcal{L}(H)$ such that

$$
T=\int_{\Delta} \widehat{T}(\delta) d F(\delta), \quad T \in \mathcal{A} .
$$

Some explanation is in order. The space $\Delta$ in Theorem 1 is the structure space of $\mathcal{A}$; it is a compact Hausdorff space and can be interpreted as the set of all nonzero, complex homomorphisms $\delta: \mathcal{A} \rightarrow \mathbb{C}$ equipped with the topology of pointwise convergence on $\mathcal{A}$. Given $T \in \mathcal{A}$, the continuous function $\widehat{T}: \Delta \rightarrow \mathbb{C}$ is defined by $\widehat{T}(\delta):=\delta(T)$, for $\delta \in \Delta$; it is called the Gelfand transform of $T$. We denote by $\mathcal{B}(\Delta)$ the Borel $\sigma$-algebra of $\Delta$, i.e. the smallest $\sigma$-algebra containing all the open subsets of $\Delta$. To say a function $F: \mathcal{B}(\Delta) \rightarrow \mathcal{L}(H)$ is a selfadjoint spectral measure means that $F(\Delta)=I$, that each operator $F(A)$, for $A \in \mathcal{B}(\Delta)$, is selfadjoint, that $F$ is multiplicative (i.e. $F(A \cap B)=F(A) F(B)$ for all $A, B \in \mathcal{B}(\Delta)$ ), and that $F$ is countably additive for the weak (equivalently, the strong) operator topology in $\mathcal{L}(H)$, that is, $F_{x, y}: A \mapsto\langle F(A) x, y\rangle$, for $A \in \mathcal{B}(\Delta)$, is a $\sigma$-additive, $\mathbb{C}$-valued

Received by the editors March 3, 1997.

1991 Mathematics Subject Classification. Primary 47A67, 47B15, 47D03, 47D25.

The support of the Deutscher Akademischer Austauschdienst (DAAD) is gratefully acknowledged by the second author.

(C)1998 American Mathematical Society 
measure for each $x, y \in H$. Since all the values $F(A)$, for $A \in \mathcal{B}(\Delta)$, are orthogonal projections, we see that $F_{x, x}(A)=\|F(A) x\|^{2}$ is actually non-negative, for each $x \in H$. To say that $F$ is Radon means that $F_{x, x}$ is a Radon measure (i.e. inner regular), for each $x \in H$; see [1, Ch. 2], for example. The selfadjointness of $F$ implies that

$$
4 F_{x, y}=F_{x+y, x+y}-F_{x-y, x-y}+i F_{x+i y, x+i y}-i F_{x-i y, x-i y}, \quad x, y \in H,
$$

and so each complex measure $F_{x, y}$ is also Radon. Since $\Delta$ is compact and $\widehat{T}$ is continuous, it is, in particular, a bounded Borel function and so the integral in (1) defines an element of $\mathcal{L}(H)$ via the standard theory of integration with respect to spectral measures; see [5, Section 12.20].

Let $S$ be a commutative semigroup with identity element (always denoted by $e$ ) and equipped with an involution $s \mapsto s^{-}$(i.e. $\left(s^{-}\right)^{-}=s$ and $(s t)^{-}=s^{-} t^{-}$for all $s, t \in S)$. A character of $S$ is any function $\rho: S \rightarrow \mathbb{C}$ satisfying $\rho(e)=1$ and $\rho\left(s t^{-}\right)=\rho(s) \overline{\rho(t)}$ for all $s, t \in S$. The set of all characters of $S$ is denoted by $S^{*}$; it is a completely regular space when equipped with the topology of pointwise convergence inherited from $\mathbb{C}^{S}$. If $H$ is a Hilbert space, then a map $\mathcal{U}: S \rightarrow \mathcal{L}(H)$ is called a *-representation if $\mathcal{U}(e)=I$ and $\mathcal{U}\left(s t^{-}\right)=\mathcal{U}(s) \mathcal{U}(t)^{*}$ for all $s, t \in S$.

Theorem 2. Let $S$ be a commutative semigroup with identity and an involution, and let $\mathcal{U}: S \rightarrow \mathcal{L}(H)$ be a $*$-representation. Then there exists a unique selfadjoint, Radon spectral measure $E: \mathcal{B}\left(S^{*}\right) \rightarrow \mathcal{L}(H)$ which has compact support, such that

$$
\mathcal{U}(s)=\int_{S^{*}} \hat{s}(\rho) d E(\rho), \quad s \in S .
$$

Again some explanation is needed. The support of any spectral measure $E: \mathcal{B}\left(S^{*}\right)$ $\rightarrow \mathcal{L}(H)$, denoted by $\operatorname{supp}(E)$, is defined to be the closed subset of $S^{*}$ given by $\overline{\bigcup_{x \in H} \operatorname{supp}\left(E_{x, x}\right)}$, where $\operatorname{supp}\left(E_{x, x}\right)$ is the usual support of a non-negative Radon measure [1, p. 22]. Finally, given $s \in S$, the function $\hat{s}: S^{*} \rightarrow \mathbb{C}$ is defined by $\hat{s}(\rho):=\rho(s)$, for $\rho \in S^{*}$. Since $\hat{s}$ is continuous and $\operatorname{supp}(E)$ is compact, the spectral integral on the right-hand-side of (2) exists and is an element of $\mathcal{L}(H)$.

A version of Theorem 2 is formulated in [2, Theorem 2.6] where it is indicated that the proof is a combination of an integral representation theorem for exponentially bounded, positive definite functions on semigroups (now-a-days referred to as the Berg-Maserick theorem) [2, Theorem 2.1], together with the method of proof given for an earlier version of Theorem 2 formulated for uniformly bounded representations $\mathcal{U}$ [3, Theorem 3.2]. An examination of that proof (i.e. when $\mathcal{U}$ is uniformly bounded) shows that an essential ingredient is the use of the theory of abelian $C^{*}$-algebras (cf. Section 2 of [3]) together with some more general Banach algebra arguments (cf. [3, p. 501]).

The aim of this note is to highlight the fact, contrary to the line of argument suggested above, that Theorems 1 and 2 are actually "independent" of one another. That is, we present a (new) proof of Theorem 2 which uses neither GelfandNaimark theory nor any Banach algebra arguments (either directly or indirectly). We then use Theorem 2 to establish Theorem 1, thereby giving a new proof of the Gelfand-Naimark theorem for abelian $C^{*}$-algebras. Conversely, via a quite different argument than that suggested by Berg and Maserick, we show that Theorem 2 also follows from Theorem 1 .

In order to prove Theorem 2 we require a few preliminaries. A function $\varphi: S \rightarrow$ $\mathbb{C}$ is called positive definite if $\sum_{j, k=1}^{n} c_{j} \bar{c}_{k} \varphi\left(s_{j} s_{k}^{-}\right) \geq 0$ for all choices of $n \in \mathbb{N}$, 
$\left\{s_{1}, \ldots, s_{n}\right\} \subseteq S$ and $\left\{c_{1}, \ldots, c_{n}\right\} \subseteq \mathbb{C}$. In particular, every character $\rho \in S^{*}$ is positive definite.

A function $\alpha: S \rightarrow[0, \infty)$ satisfying $\alpha(e)=1$ is called an absolute value if $\alpha\left(s^{-}\right)=\alpha(s)$ and $\alpha(s t) \leq \alpha(s) \alpha(t)$ for all $s, t \in S$. A function $f: S \rightarrow \mathbb{C}$ is called $\alpha$-bounded if there exists $C>0$ such that $|f(s)| \leq C \alpha(s)$ for all $s \in S$. If $f$ happens to be positive definite and $\alpha$-bounded, then it is possible to choose $C=\varphi(e)$. A character $\rho \in S^{*}$ is $\alpha$-bounded iff $|\rho| \leq \alpha$. Hence, the set $S^{\alpha}$ of all $\alpha$-bounded characters is a compact subset of $S^{*}$. For all of these notions and further properties we refer to $[1, \mathrm{Ch} .4]$.

The space of all non-negative Radon measures on $S^{*}$ is denoted by $M_{+}\left(S^{*}\right)$. Given any absolute value $\alpha: S \rightarrow[0, \infty)$ the subspace of $M_{+}\left(S^{*}\right)$ consisting of all Radon measures supported in the compact subset $S^{\alpha} \subseteq S^{*}$ is denoted by $M_{+}\left(S^{\alpha}\right)$. The following result is the Berg-Maserick theorem mentioned above.

Proposition 1. Let $\alpha: S \rightarrow[0, \infty)$ be an absolute value on $S$ and $\varphi: S \rightarrow \mathbb{C}$ an $\alpha$-bounded, positive definite function. Then there exists $\mu \in M_{+}\left(S^{\alpha}\right)$ such that

$$
\varphi(s)=\int_{S^{*}} \hat{s}(\rho) d \mu(\rho), \quad s \in S,
$$

and $\mu$ is unique within $M_{+}\left(S^{*}\right)$.

Remark 1. The function on the right-hand-side of (3), with domain $S$, is denoted by $\hat{\mu}$ and is called the generalized Laplace transform of $\mu$. It is important to note that the proof of Proposition 1 given in [1, Ch. 4, §2], which is based on the integral version of the Krein-Milman theorem, makes no use of any Banach algebra techniques what-so-ever.

Proof of Theorem 2. Define an absolute value $\alpha: S \rightarrow[0, \infty)$ by $\alpha(s)=\|\mathcal{U}(s)\|$ for $s \in S$. For $x \in H$ fixed define $\varphi_{x}: S \rightarrow \mathbb{C}$ by $\varphi_{x}(s)=\langle\mathcal{U}(s) x, x\rangle$, for $s \in S$. Using the fact that $\mathcal{U}$ is a $*$-representation it follows that

$$
\sum_{j, k=1}^{n} c_{j} \bar{c}_{k} \varphi_{x}\left(s_{j} s_{k}^{-}\right)=\left\|\sum_{j=1}^{n} c_{j} \mathcal{U}\left(s_{j}\right) x\right\|^{2} \geq 0
$$

for any finite sets $\left\{c_{1}, \ldots, c_{n}\right\} \subseteq \mathbb{C}$ and $\left\{s_{1}, \ldots, s_{n}\right\} \subseteq S$ and hence, $\varphi_{x}$ is positive definite. Moreover,

$$
\left|\varphi_{x}(s)\right| \leq\|\mathcal{U}(s)\| \cdot\|x\|^{2}=\|x\|^{2} \alpha(s), \quad s \in S,
$$

which shows that $\varphi_{x}$ is $\alpha$-bounded. By Proposition 1 there is a unique Radon measure $\mu_{x} \in M_{+}\left(S^{\alpha}\right)$ such that $\varphi_{x}=\hat{\mu}_{x}$.

For $x, y \in H$ define a complex Radon measure $\mu_{x, y}$ by

$$
\mu_{x, y}=\frac{1}{4}\left(\mu_{x+y}-\mu_{x-y}+i \mu_{x+i y}-i \mu_{x-i y}\right),
$$

in which case $\hat{\mu}_{x, y}=\frac{1}{4}\left(\hat{\mu}_{x+y}-\hat{\mu}_{x-y}+i \hat{\mu}_{x+i y}-i \hat{\mu}_{x-i y}\right)$. It then follows from the definition of $\hat{\mu}_{z}=\varphi_{z}$ for each $z \in H$ and a direct calculation that

$$
\hat{\mu}_{x, y}(s)=\langle\mathcal{U}(s) x, y\rangle, \quad s \in S .
$$

Fix $B \in \mathcal{B}\left(S^{*}\right)$ and define $\Psi_{B}: H \times H \rightarrow \mathbb{C}$ by $\Psi_{B}(x, y)=\mu_{x, y}(B)$ for each $x, y \in H$. It is clear from (5) and the linearity and injectivity of the map $\nu \mapsto \hat{\nu}$ on the space of compactly supported (complex) Radon measures on $S^{*}[1$, p. 96] that $\Psi_{B}$ is a sesquilinear form on $H \times H$. We proceed to show that $\Psi_{B}$ is bounded. 
Let $\left\{x_{1}, \ldots, x_{n}\right\} \subseteq H$ and $\left\{c_{1}, \ldots, c_{n}\right\} \subseteq \mathbb{C}$ be finite sets. Define a Radon measure $\nu$ on $\mathcal{B}\left(S^{*}\right)$ by $\nu=\sum_{j, k=1}^{n} c_{j} \bar{c}_{k} \mu_{x_{j}, x_{k}}$. Then, for finite sets $\left\{d_{1}, \ldots, d_{m}\right\} \subseteq \mathbb{C}$ and $\left\{t_{1}, \ldots, t_{m}\right\} \subseteq S$ we have

$$
\sum_{p, q=1}^{m} d_{p} \bar{d}_{q} \hat{\nu}\left(t_{p} t_{q}^{-}\right)=\sum_{j, k} \sum_{p, q} d_{p} \bar{d}_{q} c_{j} \bar{c}_{k}\left\langle\mathcal{U}\left(t_{p} t_{q}^{-}\right) x_{j}, x_{k}\right\rangle=\left\|\sum_{p, j} c_{j} d_{p} \mathcal{U}\left(t_{p}\right) x_{j}\right\|^{2} \geq 0,
$$

which shows that $\hat{\nu}: S \rightarrow \mathbb{C}$ is positive definite. By combining Theorem 2.5 on p. 93 and the remarks of $\S 2.10$ on p. 96 in [1] it follows that $\nu \geq 0$, i.e. $\nu \in M_{+}\left(S^{\alpha}\right)$. In particular,

$$
\sum_{j, k=1}^{n} c_{j} \bar{c}_{k} \Psi_{B}\left(x_{j}, x_{k}\right)=\sum_{j, k=1}^{n} c_{j} \bar{c}_{k} \mu_{x_{j}, x_{k}}(B)=\nu(B) \geq 0
$$

which shows that $\Psi_{B}$ is a positive definite kernel (in the sense of [1, p. 67], for example). By the Cauchy-Schwarz inequality for such kernels (cf. [1, p. 69], for example) we have $\left|\Psi_{B}(x, y)\right|^{2} \leq \Psi_{B}(x, x) \Psi_{B}(y, y)$, that is,

$$
\left|\mu_{x, y}(B)\right| \leq\left[\mu_{x, x}(B)\right]^{1 / 2}\left[\mu_{y, y}(B)\right]^{1 / 2} \leq\|x\| \cdot\|y\|, \quad x, y \in H,
$$

where the last inequality relies on the observation that

$$
\mu_{x, x}(B) \leq \mu_{x, x}\left(S^{\alpha}\right)=\int_{S^{*}} \rho(e) d \mu_{x, x}(\rho)=\hat{\mu}_{x, x}(e)=\langle\mathcal{U}(e) x, x\rangle=\|x\|^{2}
$$

for all $x \in H$. So, the sesquilinear form $\Psi_{B}$ is indeed bounded and hence, there is $E(B) \in \mathcal{L}(H)$ satisfying

$$
\langle E(B) x, y\rangle=\Psi_{B}(x, y)=\mu_{x, y}(B), \quad x, y \in H .
$$

To see that $E(B)^{*}=E(B)$ we note that

$$
\left\langle E(B)^{*} x, y\right\rangle=\langle x, E(B) y\rangle=\overline{\langle E(B) y, x\rangle}=\overline{\mu_{y, x}(B)}=\mu_{x, y}(B)=\langle E(B) x, y\rangle
$$

for all $x, y \in H$, where $\overline{\mu_{y, x}(B)}=\mu_{x, y}(B)$ follows again from the positive definiteness of the kernel $\Psi_{B}$. Furthermore, $E\left(S^{*}\right)=I$ since, by (5), we have

$$
\left\langle E\left(S^{*}\right) x, y\right\rangle=\mu_{x, y}\left(S^{*}\right)=\hat{\mu}_{x, y}(e)=\langle\mathcal{U}(e) x, y\rangle=\langle x, y\rangle, \quad x, y \in H .
$$

It is clear from (6) that $E_{x, y}=\mu_{x, y}$ is $\sigma$-additive for all $x, y \in H$. The identity (6) also shows that $E: \mathcal{B}\left(S^{*}\right) \rightarrow \mathcal{L}(H)$ is a Radon measure and that it is compactly supported since $\operatorname{supp}\left(E_{x, y}\right)=\operatorname{supp}\left(\mu_{x, y}\right) \subseteq S^{\alpha}$ for all $x, y \in H$.

To see that $E$ is multiplicative we proceed as follows. Given a Borel set $A \subseteq S^{*}$ and $x, y \in H$ define measures $\nu_{A}$ and $\lambda_{A}$ by $\nu_{A}(B)=\mu_{E(A) x, y}(B)$ and $\lambda_{A}(B)=$ $\langle E(A \cap B) x, y\rangle=\mu_{x, y}(A \cap B)$ for each $B \in \mathcal{B}\left(S^{*}\right)$. Using (5) we have

$$
\hat{\nu}_{A}(t)=\hat{\mu}_{E(A) x, y}(t)=\langle\mathcal{U}(t) E(A) x, y\rangle=\left\langle E(A) x, \mathcal{U}(t)^{*} y\right\rangle=\mu_{x, \mathcal{U}(t)^{*} y}(A)
$$

and

$$
\hat{\lambda}_{A}(t)=\int_{A} \rho(t) d \mu_{x, y}(\rho) .
$$

For a fixed $t \in S$ it is clear from (7) and (8) that the mappings $\tau_{1}: A \mapsto \hat{\nu}_{A}(t)$ and $\tau_{2}: A \mapsto \hat{\lambda}_{A}(t)$ are Radon measures on $S^{*}$, supported in $S^{\alpha}$, and so we can also calculate $\hat{\tau}_{1}$ and $\hat{\tau}_{2}$. Indeed, for $s \in S$, we have (by (5) and (7)) that

$$
\hat{\tau}_{1}(s)=\left\langle\mathcal{U}(s) x, \mathcal{U}(t)^{*} y\right\rangle=\langle\mathcal{U}(s t) x, y\rangle=\int_{S^{*}} \rho(s) \rho(t) d \mu_{x, y}(\rho) .
$$


But, from (8) it is also clear that

$$
\hat{\tau}_{2}(s)=\int_{S^{*}} \rho(s) d \tau_{2}(\rho)=\int_{S^{*}} \rho(s) \rho(t) d \mu_{x, y}(\rho) .
$$

By uniqueness of generalized Laplace transforms [1, p. 96], it follows that $\tau_{1} \equiv \tau_{2}$ as compactly supported Radon measures (for any fixed $t \in S$ ). In particular, for $A$ fixed we deduce that $\hat{\nu}_{A}(t)=\hat{\lambda}_{A}(t)$. Since this is true for each $t \in S$ it again follows that $\nu_{A}=\lambda_{A}$ as measures and hence, for any Borel set $B \subseteq S^{*}$ we have $\lambda_{A}(B)=\nu_{A}(B)$, that is,

$$
\langle E(A \cap B) x, y\rangle=\lambda_{A}(B)=\nu_{A}(B)=\langle E(B) E(A) x, y\rangle, \quad x, y \in H .
$$

So, $E$ is multiplicative.

Finally, $\hat{s}: S^{*} \rightarrow \mathbb{C}$ is continuous and hence bounded on $S^{\alpha}$, for any $s \in S$. By the theory of spectral integrals $\int_{S^{*}} \hat{s}(\rho) d E(\rho)$ exists in $\mathcal{L}(H)$. It is clear from (5) and (6) that $\mathcal{U}(s)=\int_{S^{*}} \hat{s}(\rho) d E(\rho)$, for $s \in S$, and the proof of Theorem 2 is complete.

To deduce Theorem 1 from Theorem 2 we require a further result. A commutative, unital complex algebra $\mathcal{A}$ with an involution (see Definitions 10.1 and 11.14 in [5]) always induces a commutative semigroup with involution, namely let $S=\mathcal{A}$ and consider $\mathcal{A}$ just with respect to its given multiplication and involution. We then define $S^{\circledast}:=\left\{\rho \in S^{*}: \rho\right.$ is linear $\}$ and, given any absolute value $\alpha: S \rightarrow[0, \infty)$, define $S^{\circledR}:=S^{\alpha} \cap S^{\circledast}$. The following result can be found in [4, Corollary 1]; we stress again that its proof does not use any Banach algebra methods.

Proposition 2. Let $S$ be the semigroup induced by a unital, commutative complex algebra with an involution. Let $\alpha: S \rightarrow[0, \infty)$ be an absolute value. If $\varphi: S \rightarrow \mathbb{C}$ is a positive definite, $\alpha$-bounded function which is linear on $S$, then the unique Radon measure $\mu \in M_{+}\left(S^{\alpha}\right)$ satisfying $\hat{\mu}=\varphi$ has its support in $S @$.

Proof of Theorem 1. Let $A \subseteq \mathcal{L}(H)$ be an abelian $C^{*}$-algebra. Let $S=\mathcal{A}$, considered as a semigroup with respect to the multiplication in $\mathcal{A}$ and with the involution from $\mathcal{A}$. By Theorem 2 there exists a unique, selfadjoint Radon spectral measure $E: \mathcal{B}\left(S^{*}\right) \rightarrow \mathcal{L}(H)$ with compact support satisfying

$$
T=\int_{S^{*}} \widehat{T}(\rho) d E(\rho), \quad T \in S
$$

we have used the representation $\mathcal{U}(T)=T$, for $T \in S$. From the proof of Theorem 2 , where the absolute value $\alpha: S \rightarrow[0, \infty)$ used is $\alpha(T)=\|\mathcal{U}(T)\|=\|T\|$, for $T \in S$, we have that $\operatorname{supp}(E) \subseteq S^{\alpha}$. Now, for $x \in H$ fixed, the positive definite and $\alpha$-bounded function $\varphi_{x}: S \rightarrow \mathbb{C}$ defined in the proof of Theorem 2 (i.e. $\varphi_{x}(T)=$ $\langle\mathcal{U}(T) x, x\rangle=\langle T x, x\rangle$, for $T \in S)$ is clearly linear on $S$. Then Proposition 2 implies that the unique measure $\mu_{x} \in M_{+}\left(S^{\alpha}\right)$ satisfying $\hat{\mu}_{x}=\varphi_{x}$ has its support in $S @$. Since $E_{x, x}=\mu_{x}$ also $\operatorname{supp}(E) \subseteq S @$. But, if $\rho \in S @$, then

$$
|\rho(T)| \leq \alpha(T)=\|T\|, \quad T \in \mathcal{A},
$$

which implies that $\|\rho\|=1$. So, $S @$ consists of all algebra homomorphisms $\rho$ : $S(=\mathcal{A}) \rightarrow \mathbb{C}$ of norm 1 . It is part of the definition that each semigroup character $\rho \in S^{*}$ must satisfy $\rho\left(s^{-}\right)=\overline{\rho(s)}$ which, in the present setting, becomes $\rho\left(T^{*}\right)=\overline{\rho(T)}$. But, elements of the structure space $\Delta$ of $\mathcal{A}$ automatically satisfy this condition [5, Theorem 11.18]. Hence, $S @$ is precisely $\Delta$ and (9), which is actually an integral over $S @=\Delta$, reduces to (1). 
In conclusion we indicate how Theorem 2 also follows from Theorem 1. So, let $S$ and $\mathcal{U}: S \rightarrow \mathcal{L}(H)$ be as in Theorem 2. Define $\mathcal{A} \subseteq \mathcal{L}(H)$ to be the $C^{*}$-algebra generated by the $*$-closed, commutative family of operators $\mathcal{M}=\{\mathcal{U}(s): s \in S\}$. Of course, $\mathcal{A}$ is the operator norm closure in $\mathcal{L}(H)$ of the linear span of $\mathcal{M}$. Denote the structure space of $\mathcal{A}$ by $\Delta$. For each $\delta \in \Delta$ define $\tilde{\delta}: S \rightarrow \mathbb{C}$ by $\tilde{\delta}(s):=$ $\delta(\mathcal{U}(s)), s \in S$. From the homomorphism properties of $\delta$ and the fact that $\mathcal{U}$ is a *-representation it is easily seen that $\tilde{\delta} \in S^{*}$; the property $\tilde{\delta}\left(s^{-}\right)=\overline{\tilde{\delta}(s)}$ again follows from the comments at the end of the previous paragraph. So, we can define $\Lambda: \Delta \rightarrow S^{*}$ by $\Lambda(\delta):=\tilde{\delta}$, for $\delta \in \Delta$, in which case $\hat{s} \circ \Lambda=(\mathcal{U}(s))^{\wedge}$ as functions on $\Delta$, for each $s \in S$. If $\Lambda\left(\delta_{1}\right)=\Lambda\left(\delta_{2}\right)$, then $\delta_{1}(\mathcal{U}(s))=\delta_{2}(\mathcal{U}(s))$ for all $s \in S$ and hence, $\delta_{1}$ and $\delta_{2}$ agree on the linear span of $\mathcal{M}$. Since the span of $\mathcal{M}$ is dense in $\mathcal{A}$ and both $\delta_{1}$ and $\delta_{2}$ are continuous on $\mathcal{A}$, it follows that $\delta_{1}=\delta_{2}$. This shows that $\Lambda$ is injective. Since $\Delta$ (resp. $S^{*}$ ) is equipped with the pointwise convergence topology on $\mathcal{A}$ (resp. $S$ ), it is clear that $\Lambda$ is continuous. Then the compactness of $\Delta$ ensures that $\Lambda$ is a topological homeomorphism of $\Delta$ onto its range $K:=\Lambda(\Delta) \subseteq S^{*}$. By Theorem 1 there is a unique selfadjoint Radon spectral measure $F: \mathcal{B}(\Delta) \rightarrow \mathcal{L}(H)$ satisfying

$$
T=\int_{\Delta} \widehat{T}(\delta) d F(\delta), \quad T \in \mathcal{A} .
$$

Define $E: \mathcal{B}\left(S^{*}\right) \rightarrow \mathcal{L}(H)$ by $E(A)=F\left(\Lambda^{-1}(A)\right)$, for each $A \in \mathcal{B}\left(S^{*}\right)$, in which case $E$ is a compactly supported $(\operatorname{as} \operatorname{supp}(E) \subseteq K)$, selfadjoint Radon spectral measure such that

$$
\mathcal{U}(s)=\int_{\Delta}(\mathcal{U}(s))^{\wedge}(\delta) d F(\delta)=\int_{S^{*}} \hat{s}(\rho) d E(\rho), \quad s \in S
$$

see [5, Theorem 13.28] for the last equality.

Suppose now that $G: \mathcal{B}\left(S^{*}\right) \rightarrow \mathcal{L}(H)$ is another compactly supported, selfadjoint Radon spectral measure satisfying

$$
\mathcal{U}(s)=\int_{S^{*}} \hat{s}(\rho) d G(\rho), \quad s \in S .
$$

It follows that $\int_{S^{*}} \hat{s}(\rho) d G_{x, x}(\rho)=\int_{S^{*}} \hat{s}(\rho) d E_{x, x}(\rho)$ for all $s \in S$ and $x \in H$, that is, $\widehat{G}_{x, x}=\widehat{E}_{x, x}$ for all $x \in H$. Since both $G_{x, x}$ and $E_{x, x}$ are compactly supported elements of $M_{+}\left(S^{*}\right)$, it follows from the uniqueness of generalized Laplace transforms that $E_{x, x}=G_{x, x}$ for all $x \in H$. Then the selfadjointness of both $E$ and $G$ and the polarization identity imply that $E_{x, y}=G_{x, y}$ for all $x, y \in H$. In particular, it follows that $E(A)=G(A)$ for all $A \in \mathcal{B}\left(S^{*}\right)$, that is, $E=G$. Hence, the measure $E$ satisfying (10) is unique and the proof is complete.

Remark 2. From the proof of Theorem 2 it is obvious that if all the measures $E_{x, x}=\mu_{x}$, for $x \in H$, concentrate on a subset $A \subseteq S^{*}$, then so does $E$. As a consequence we obtain Stone's theorem for $S$ a locally compact abelian group (which is a semigroup with the special involution $s^{-}:=s^{-1}$ ), and where $\mathcal{U}$ is assumed to be weak operator continuous, i.e. $s \mapsto\langle\mathcal{U}(s) x, x\rangle$ is continuous for each $x \in H$. Then by Bochner's theorem all the measures $\mu_{x}$ live on the dual group (by definition the set of all continuous characters), and so therefore does $E$. 


\section{REFERENCES}

1. C. Berg, J. P. R. Christensen and P. Ressel, Harmonic analysis on semigroups, Theory of positive definite and related functions, Graduate Texts in Mathematics Vol. 100, SpringerVerlag, New York-Berlin-Heidelberg-Tokyo, 1984. MR 86b:43001

2. C. Berg and P. H. Maserick, Exponentially bounded positive definite functions, Illinois J. Math. 28 (1984), 162-179. MR 85i:43012

3. P. H. Maserick, Spectral theory of operator-valued transformations, J. Math. Anal. Appl. 41 (1973), 497-507. MR 49:7828

4. P. Ressel, Integral representations on convex semigroups, Math. Scand. 61 (1987), 93-111. MR 89e: 43012

5. W. Rudin, Functional analysis, McGraw Hill Book Co., New York-San Francisco-St. Louis, 1973. MR 51:1315

Math.-Geogr. Fakultät, Katholische Universität Eichstätt, D-85071 Eichstätt, Germany

School of Mathematics, University of New South Wales, Sydney, New South Wales, 2052 Australia 